\title{
The role of anti-elastin antibodies in a mouse model of asthma
}

\author{
Tomoshige Wakayama, ${ }_{1}$ Kumiya Sugiyama, ${ }^{1,2}$ Shingo Tokita, ${ }^{1}$ Hajime Arifuku, ${ }^{1}$ Naotatsu Otsuji, ${ }^{1}$ Kei Sugitate, ${ }^{1}$ \\ Takayoshi Owada, ${ }^{1}$ Kenya Koyama, ${ }^{1}$ Hirokuni Hirata, ${ }^{1}$ Masafumi Arima, ${ }^{3}$ Yasutsugu Fukushima ${ }^{1}$
}

\begin{abstract}
Background: The role of anti-elastin antibody $(\mathrm{Ab})$ in the lung is unclear, although they may be involved in chronic obstructive pulmonary disease (COPD). Recently, increased anti-elastin Ab levels were reported in asthma.

Objective: To elucidate the role of anti-elastin $\mathrm{Ab}$ in asthma, we created a murine asthma model. Anti-elastin Ab in the airway was neutralized by intratracheal administration of elastin peptide, and the inhibitory effects of anti-elastin Ab on airway remodeling were evaluated.

Methods: BALB/c mice were immunized with ovalbumin (OVA) on days 0 and 14. After immunization, the mice received booster OVA via inhalation twice per week for 9 weeks, and bronchoalveolar lavage fluid (BALF) and lung tissues were evaluated.

Results: In lung tissues, airway remodeling occurred after 9 weeks of OVA sensitization. Peak levels of anti-elastin Ab and eosinophils in BALF were detected after 3 weeks of OVA sensitization. Anti-elastin Ab and eosinophil levels in BALF were significantly reduced after 3 weeks by the neutralization of anti-elastin Ab. Peak transforming growth factor- $\beta 1$ levels in BALF were detected at 3 weeks after OVA sensitization and were significantly reduced by the neutralization of anti-elastin Ab. Airway remodeling in lung tissues was also significantly inhibited by the neutralization of anti-elastin $\mathrm{Ab}$.
\end{abstract}

Conclusion: In our murine asthma model, anti-elastin Ab was recruited to the airway by OVA-induced allergic inflammation. Airway remodeling was inhibited by the neutralization of anti-elastin Ab. Anti-elastin Ab may contribute to the progression of airway remodeling.

Key words: Anti-elastin antibodies, Airway remodeling, Asthma, Eosinophils, TGF- $\beta$

From:

${ }^{1}$ Department of Respiratory Medicine and Clinical Immunology Dokkyo Medical University, Saitama Medical Center, Saitama, Japan

${ }^{2}$ National Hospital Organization, Utsunomiya National Hospital,

Tochigi, Japan

Department of Rheumatology, Dokkyo Medical University, Tochigi, Japan

\section{Introduction}

The role of anti-elastin antibody $(\mathrm{Ab})$ in respiratory diseases has been discussed mainly in chronic obstructive pulmonary disease (COPD). High levels of anti-elastin $\mathrm{Ab}$, as an autoantibody, have been detected in the plasma of patients with severe emphysema, ${ }^{1-3}$ and anti-elastin IgM was increased by smoking in a mouse model of COPD. ${ }^{4}$ However, in another study, it was reported that anti-elastin $\mathrm{Ab}$ was decreased by smoking and not increased in patients with COPD, and smoke exposure in human participants suppressed the production of anti-elastin $\mathrm{Ab} .^{5}$ Therefore, the relationship

\section{Corresponding author:}

Kumiya Sugiyama

Department of Respiratory Medicine and Clinical Immunology

Dokkyo Medical University Saitama Medical Center

2-1-50 Minami-Koshigaya, Koshigaya, Saitama 343-8555, Japan

E-mail: sugiyama@dokkyomed.ac.jp

between anti-elastin $\mathrm{Ab}$ and COPD remains unclear. Meanwhile, a few studies have reported a relationship between asthma and anti-elastin Ab. To find useful biomarkers for the diagnosis of either asthma or COPD, we evaluated anti-elastin $\mathrm{Ab}$ in the sputum of patients with asthma and COPD. In our study, anti-elastin $\mathrm{Ab}$ in sputum was elevated in patients with asthma but not COPD. ${ }^{6}$ However, we could not determine why anti-elastin $\mathrm{Ab}$ was elevated in the sputum of patients with asthma, and research on the relationship between asthma and anti-elastin $\mathrm{Ab}$ is scarce. 
We hypothesized two possible mechanisms for the elevation of anti-elastin Ab in asthma. First, anti-elastin Ab may inhibit airway remodeling. Elastin is a collagen fiber that contributes to airway remodeling. Elastin may be reduced by anti-elastin $\mathrm{Ab}$, thereby inhibiting airway remodeling. In the second theory, anti-elastin $\mathrm{Ab}$ may be a factor in the progression of airway remodeling. In addition to eosinophilic airway inflammation, additional airway inflammation may be caused by antigen-antibody reactions, and airway remodeling may progress.

To elucidate the role of anti-elastin Ab in asthma, we created a mouse model of asthma and evaluated the production of anti-elastin $\mathrm{Ab}$. Then, we neutralized anti-elastin $\mathrm{Ab}$ in the airway by intratracheal administration of elastin peptide, evaluated the inhibitory effects on airway remodeling, and speculated about the role of anti-elastin $\mathrm{Ab}$ based on those results.

\section{Methods \\ Animals}

Female BALB/c mice (Japan SLC, Inc., Shizuoka, Japan) were used at 8 weeks of age.

\section{Antibodies and reagents}

The following drugs and chemicals were purchased: ovalbumin (OVA; Fujifilm Wako Pure Chemical Co., Ltd., Osaka, Japan), aluminum hydroxide (Thermo Fisher Scientific, Waltham, MA), saline solution (Otsuka Pharmaceutical, Tokyo, Japan), mouse lung elastin peptide (Elastin Products Company, Inc., Owensville, MO), rabbit anti-mouse elastin polyclonal Ab (ab217356; Abcam, Cambridge, UK), soluble bovine elastin (Elastin Products Company), mouse anti-mouse elastin monoclonal Ab (SC-58756; Santa Cruz Biotechnology, Dallas, TX), goat anti-mouse IgG F(ab')2 Ab-horseradish peroxidase (SAB3701015; Sigma-Aldrich, St. Louis, MO), mouse anti- $\alpha$-smooth muscle actin monoclonal Ab (ab1814: Abcam), Protein Block Serum-Free (Agilent Technologies, Inc.,
Santa Clara, CA), mouse transforming growth factor (TGF)- $\beta 1$ enzyme-linked immunosorbent assay (ELISA) kit (KE10005; Proteintech Group Inc., Rosemont, IL), o-phenylenediammonium dichloride (Fujifilm Wako Pure Chemical Co., Ltd.), May-Grunwald's stain solution (Muto Pure Chemicals Co., Ltd., Tokyo, Japan), microscopy Giemsa's-azur-eosin methylene blue solution (Merck KGaA, Darmstadt, Germany), Histofine $^{\varpi}$ Simple Stain Mouse and Histofine ${ }^{\circledast}$ MOUSESTAIN Kit (Nichirei Biosciences, Inc., Tokyo, Japan), Schiff's reagent for microscopy (109033; Merck KGaA), DAB+ substrate buffer (Agilent Technologies, Inc.), and $\mathrm{DAB}+$ Chromogen (Agilent Technologies, Inc.).

\section{Study design}

Mouse models of asthma were established from OVA-sensitized mice according to previously reported methods with some modifications. ${ }^{7-11}$ Briefly, BALB/c mice were injected intraperitoneally with $50 \mu \mathrm{g}$ OVA in $4 \mathrm{mg}$ aluminum hydroxide on days 0 and 14 (Figure 1A). To establish an allergic inflammation model of asthma, sensitivity was boosted by 20 -min inhalation of $1 \mathrm{mg} / \mathrm{mL}$ OVA twice per week from day $21 \mathrm{un}$ til day 76 (asthma group). A normal control (NC) group received saline instead of OVA. For inhalation, the mice were placed in sealed chambers and drugs were aerosolized using an ultrasonic nebulizer (Omron, Kyoto, Japan). The mice were sacrificed, and bronchoalveolar lavage fluid (BALF) and lung tissues were collected every week from day 28 (1 week after sensitization) until day 84 ( 9 weeks after sensitization). To evaluate the effects on neutralized anti-elastin $\mathrm{Ab}, 50 \mu \mathrm{L}$ mouse elastin peptide solution $(40 \mu \mathrm{g} / \mathrm{mL}$ diluted in saline) was administered intratracheally weekly from day 22 until day 77 using aerosol administration equipment (FMJ-250 High Pressure Syringe and MicroSprayer ${ }^{\circledR}$ Aerosolizer - Model IA1C-R; Penn Century, Inc., Wyndmoor, PA) (Figure 1B).

The study was approved by the Animal Care and Use Committee of Dokkyo Medical University (No. 1045).

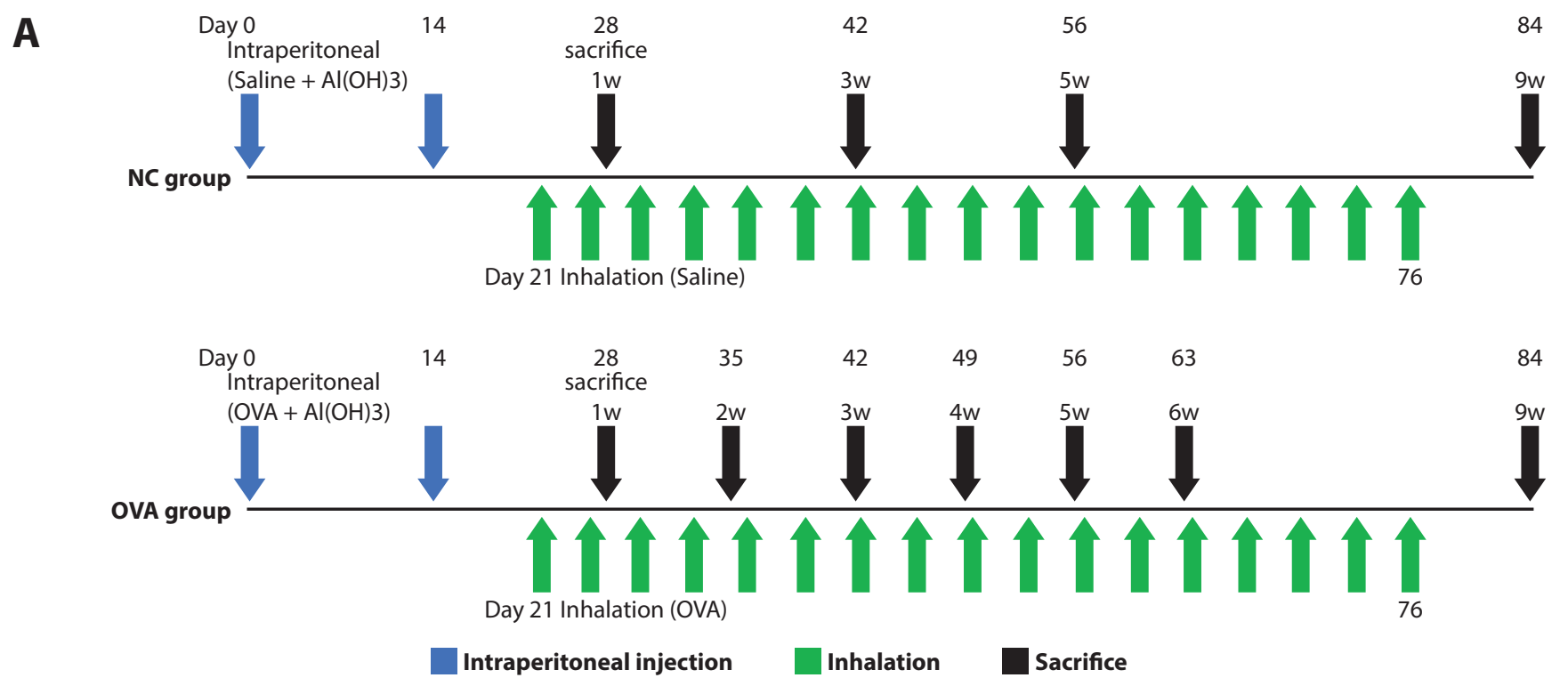

Figure 1. Study protocol. 

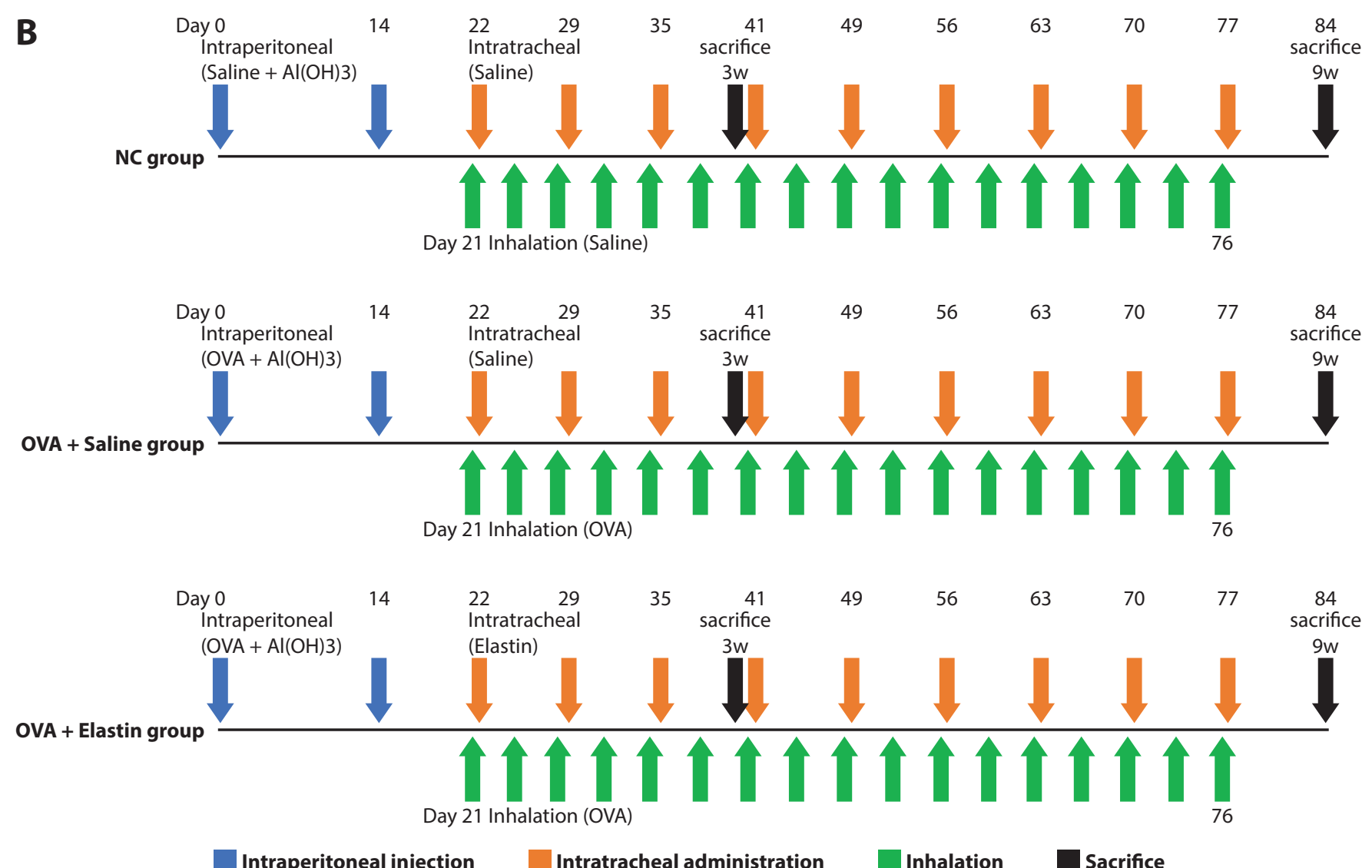

Figure 1. (Continued)

\section{Preparations for cytological and histological analyses}

For cytological analysis, BALF was prepared by centrifugation for $10 \mathrm{~min}$ at $400 \times \mathrm{g}$ and supernatants were frozen at $-80^{\circ} \mathrm{C}$ for the biological assays. A cell pellet was added to $1 \mathrm{~mL}$ saline solution, and the total number of cells in BALF was counted. In addition, a $600-\mu \mathrm{L}$ cell pellet solution was prepared by cytospin for $3 \mathrm{~min}$ at $500 \mathrm{rpm}$, the slides were treated with Giemsa staining, and the number of eosinophils was counted.

For histological evaluation, the lungs were removed, fixed in $10 \%$ formalin neutral buffer solution, embedded in paraffin, and sliced into $3-\mu \mathrm{m}$-thick sections. Elastin immunohistochemistry was performed to evaluate the expression of elastin in mouse lung tissues. Briefly, anti-mouse elastin polyclon$\mathrm{al} \mathrm{Ab}$ was incubated with mouse lung tissues overnight after blocking by Protein Block Serum-Free for $30 \mathrm{~min}$. Mouse lung tissues were then washed and subjected to Histofine ${ }^{\circ}$ Simple Stain Mouse. To detect the collagen band, the sections were stained using the elastic-Masson method. Briefly, the sections were washed and stained with resorcin-fuchsin stain, Weigert's iron hematoxylin stain, and one-step Masson's triple solution, which includes Chromotrope $2 \mathrm{R}$, light green SF, and tungstophosphoric acid, in that order. To evaluate peribronchial smooth muscle, the sections were incubated with primary mouse anti- $\alpha$-smooth muscle actin $\mathrm{Ab}$ and then stained with a Histofine ${ }^{\bullet}$ MOUSESTAIN Kit. To detect goblet cells, periodic acid-Schiff staining was performed using cold Schiff reagent after pretreatment with $1 \%$ periodic acid solution. Finally, the sections were stained with Mayer's hematoxylin.

\section{Measurement of anti-elastin Ab, TGF- $\beta 1$, and eosinophils in $B A L F$}

The anti-mouse elastin $\mathrm{Ab}$ quantification assay was performed using a modified ELISA protocol. ${ }^{3}$ Briefly, mouse lung elastin peptide was used to coat ELISA plates. After incubation and washing, BALF samples were diluted and incubated. After further washing, anti-mouse $\operatorname{IgG~F}\left(\mathrm{ab}^{\prime}\right) 2$ Ab-horseradish peroxidase was added and incubated. The plates were washed again, o-phenylenediamine dichloride was added, and the optical density of the individual wells was determined. Mouse anti-mouse elastin monoclonal $\mathrm{Ab}$ was used to generate the standard curve. The TGF- $\beta 1$ quantification assay was performed using a mouse TGF- $\beta 1$ ELISA Kit.

\section{Imaging and statistical analyses}

To measure the area for histological evaluation, Image ${ }^{\circ}$ version $1.53 \mathrm{e}$ (National Institutes of Health, Bethesda, MD) was used. Briefly, the target color was replaced with red by Image ${ }^{\circ}$. After we determined that it was correctly replaced, the area replaced with red was measured automatically by Image $J^{\circ}$. The whole field was set as $100 \%$, and the percentage of the red area was determined. All statistical analysis was performed using Microsoft $^{\circ}$ Excel $^{\circ} 2016$ MSO (Microsoft Corp., Redmond, WA) and JMP ${ }^{\star}$ Pro version 11.0.0 (SAS Institute, Cary, NC) statistical software. The obtained results were examined by Student's $t$-test. A $P$ value of $<0.05$ was considered statistically significant. The results are expressed as the mean \pm standard deviation. 


\section{Results}

Location of elastin in the mouse model of asthma

Elastin was significantly increased under the basement membrane in the airway at 9 weeks after OVA sensitization $(7.13 \pm 3.51 \%$ per field) compared with no OVA sensitization $(2.20 \pm 0.35 \%$ per field) ( $n=3, P<0.01)$ (Figure 2). Moreover, airway remodeling was present at 9 weeks after OVA sensitization.

\section{Levels of anti-elastin Ab after OVA sensitization}

The levels of anti-elastin $\mathrm{Ab}$ in BALF are shown in Figure 3A. The peak level was at 3 weeks after OVA sensitization (week 1, $165.1 \pm 2.9 \mathrm{ng} / \mathrm{mL}$; week 2, $262.5 \pm 58.9 \mathrm{ng} / \mathrm{mL}$; week 3, $714.4 \pm 244.2 \mathrm{ng} / \mathrm{mL}$; week 4, $380.8 \pm 60.8 \mathrm{ng} / \mathrm{mL}$; week 5 , $281.8 \pm 95.8 \mathrm{ng} / \mathrm{mL}$; week $6,341.5 \pm 35.1 \mathrm{ng} / \mathrm{mL}$; and week $9,446.8 \pm 79.6 \mathrm{ng} / \mathrm{mL})$. Significant differences were observed at weeks 1, 2, 4, 5, and 6 compared with 3 weeks after OVA sensitization. The levels of anti-elastin $\mathrm{Ab}$ in the $\mathrm{NC}$ group were significantly lower than those of the OVA group at the corresponding time points (week 1, $0.0 \pm 0 \mathrm{ng} / \mathrm{mL}$; week 3 , $0.0 \pm 0.0 \mathrm{ng} / \mathrm{mL}$; week 5, $0.0 \pm 0.0 \mathrm{ng} / \mathrm{mL}$; and week 9, $42.4 \pm$ $7.5 \mathrm{ng} / \mathrm{mL})$.

The peak level of eosinophils in BALF is shown in Figure 3B. The peak level was detected at 3 weeks after OVA sensitization, as was that of anti-elastin $\mathrm{Ab}$ (week 1, $3.8 \pm$ $1.8 \times 10^{4}$ cells/mouse; week $2,17.7 \pm 6.3 \times 10^{4}$ cells $/$ mouse; week 3, $58.9 \pm 19.7 \times 10^{4}$ cells/mouse; week $4,3.0 \pm 0.7 \times$ $10^{4}$ cells/mouse; week $5,5.3 \pm 1.0 \times 10^{4}$ cells/mouse; week $6,3.7 \pm 1.6 \times 10^{4}$ cells/mouse; and week 9, $3.6 \pm 1.7 \times 10^{4}$ cells/mouse). Significant differences were observed between week 3 and weeks 1, 2, 4, 5, 6, and 9 after OVA sensitization.

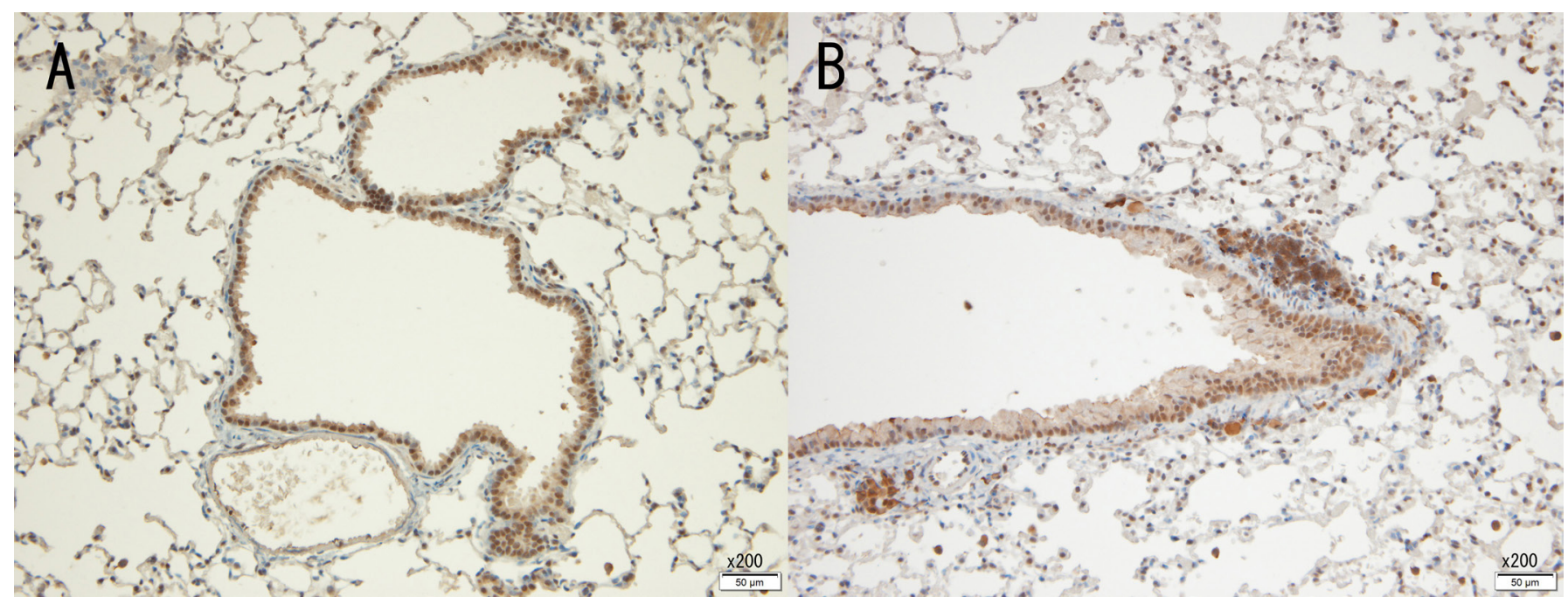

Figure 2. Locations of elastin in lung tissue. Elastin was more strongly expressed under the basement membrane around the airway after 9 weeks of OVA sensitization (B) compared with no OVA sensitization (A).

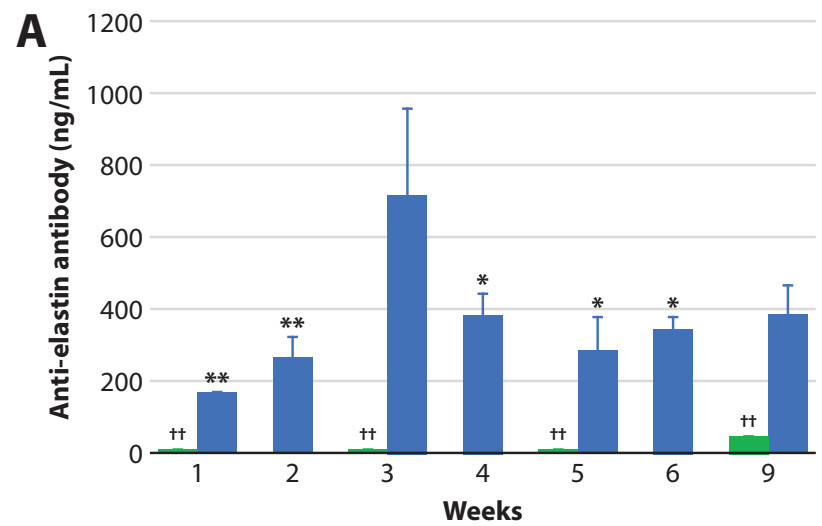

NC group
B

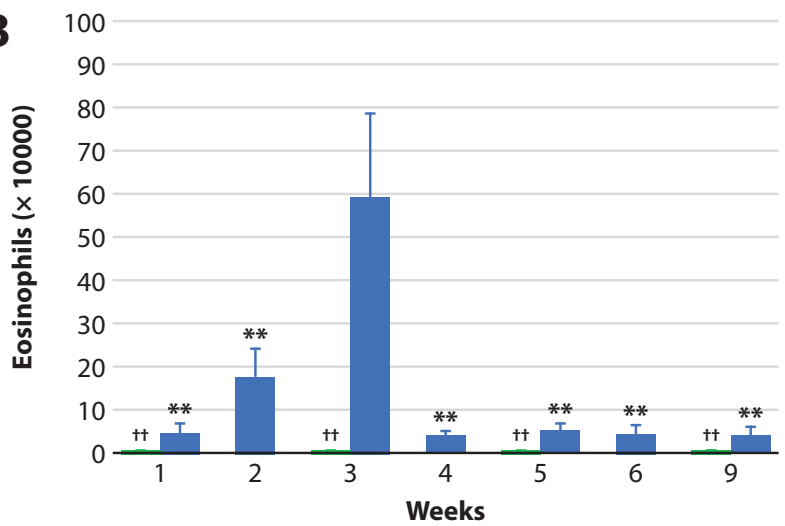

OVA group

Figure 3. Relationships between OVA sensitization and the levels of anti-elastin Ab or eosinophils in BALF. (A) Anti-elastin Ab levels were significantly increased at 3 weeks after OVA sensitization (vs. weeks 1 and $2, P<0.01$; vs. weeks 4 , 5, and 6, $P<0.05$ ). (B) Eosinophils peaked at 3 weeks after OVA sensitization and were significantly increased at week 3 after OVA sensitization compared with all other OVA sensitization time points $(P<0.01)$. The number of mice at each time point was 4 for the OVA group and 2 for the NC group. ${ }^{\star} P<0.05$ vs. week $3,{ }^{\star \star} P<0.01$ vs. week $3,{ }^{\dagger \dagger} P<0.01$ vs. the OVA group at the corresponding time points. 
A

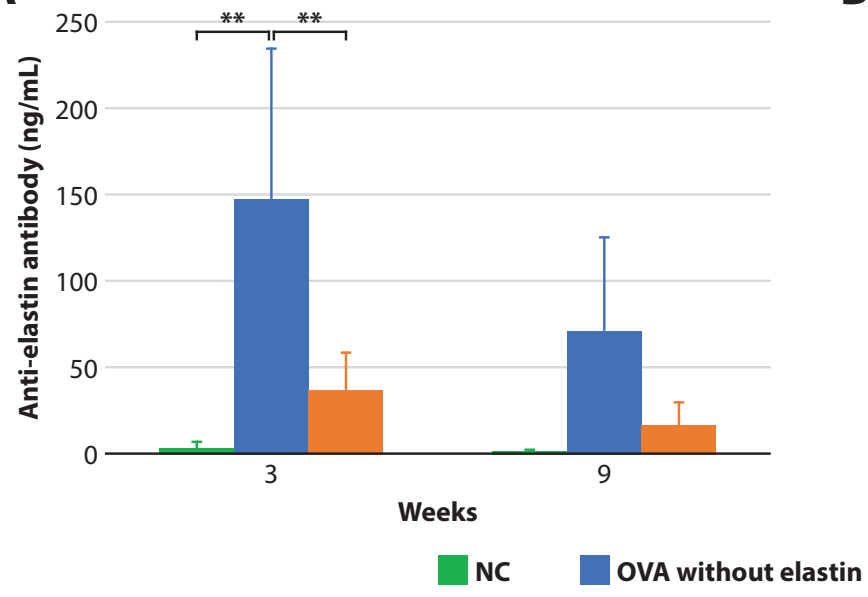

B

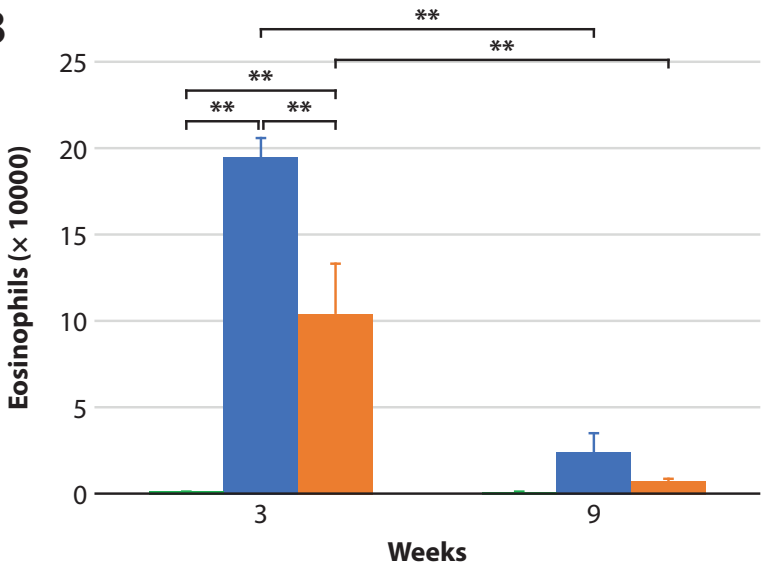

OVA with elastin

Figure 4. Neutralization effects of anti-elastin Ab after intratracheal elastin administration. (A) Anti-elastin Ab levels at 3 weeks after OVA sensitization were significantly increased $(P<0.01)$ and were significantly reduced by the neutralization of anti-elastin $\mathrm{Ab}$ following elastin intratracheal administration $(P<0.01)$; however, there were no significant differences between the groups at week 9. (B) Eosinophils were significantly reduced by the neutralization of anti-elastin Ab following elastin intratracheal administration at week 3 after OVA sensitization $(P<0.01)$. The number of mice was 3 for the NC group and 5 for the OVA groups. ${ }^{* *} P<0.01$ vs. week 3 .

The levels of eosinophils were significantly lower in the NC group than in the OVA group in the corresponding time points (week 1, $0.0 \pm 0.0 \times 10^{4}$ cells/mouse; week $3,0.0 \pm 0.0$ $\times 10^{4}$ cells/mouse; week $5,0.0 \pm 0.0 \times 10^{4}$ cells/mouse; and week $9,0.0 \pm 0.0 \times 10^{4}$ cells/mouse).

Inhibitory effects of elastin peptide intratracheal administration on anti-elastin $A b$ and eosinophils

The neutralization effects of elastin peptide intratracheal administration on anti-elastin $\mathrm{Ab}$ are shown in Figure 4. At 3 weeks after OVA sensitization, anti-elastin $\mathrm{Ab}$ levels were significantly reduced by elastin peptide intratracheal administration (Figure 4A) compared with the no-elastin-peptide groups. However, there were no significant differences at week 9 (week 3: NC $2.3 \pm 4.0 \mathrm{ng} / \mathrm{mL}$, without elastin peptide 146.3 $\pm 88.0 \mathrm{ng} / \mathrm{mL}$, with elastin peptide $35.9 \pm 22.2 \mathrm{ng} / \mathrm{mL}$; week 9: NC $0.0 \pm 0.0 \mathrm{ng} / \mathrm{mL}$, without elastin peptide $70.4 \pm 54.7 \mathrm{ng} /$ $\mathrm{mL}$, with elastin peptide $15.4 \pm 14.1 \mathrm{ng} / \mathrm{mL}$ ).

Eosinophil levels after the neutralization of anti-elastin $\mathrm{Ab}$ are shown in Figure 4B. Eosinophil levels in BALF were significantly reduced by elastin peptide intratracheal administration at week 3 after OVA sensitization compared with the no-elastin-peptide groups (week 3: NC $0.0 \pm 0.0 \times 10^{4}$ cells/ mouse, without elastin peptide $19.1 \pm 1.5 \times 10^{4}$ cells/mouse, with elastin peptide $10.1 \pm 3.0 \times 10^{4}$ cells/mouse; week 9: NC $0.0 \pm 0.0 \times 10^{4}$ cells/mouse, without elastin peptide $2.4 \pm 1.2$ $\times 10^{4}$ cells/mouse, with elastin peptide $0.6 \pm 0.4 \times 10^{4}$ cells/ mouse).

Inhibitory effects of neutralizing anti-elastin Ab on TGF- $\beta 1$ and airway remodeling

The levels of TGF- $\beta 1$ after the neutralization of anti-elastin $\mathrm{Ab}$ are shown in Figure 5. TGF- $\beta 1$ levels in BALF were significantly reduced by elastin peptide intratracheal administration at 3 weeks after OVA sensitization, but there were no significant differences at week 9 (week 3: NC $4.8 \pm 8.4 \mathrm{pg} / \mathrm{mL}$,

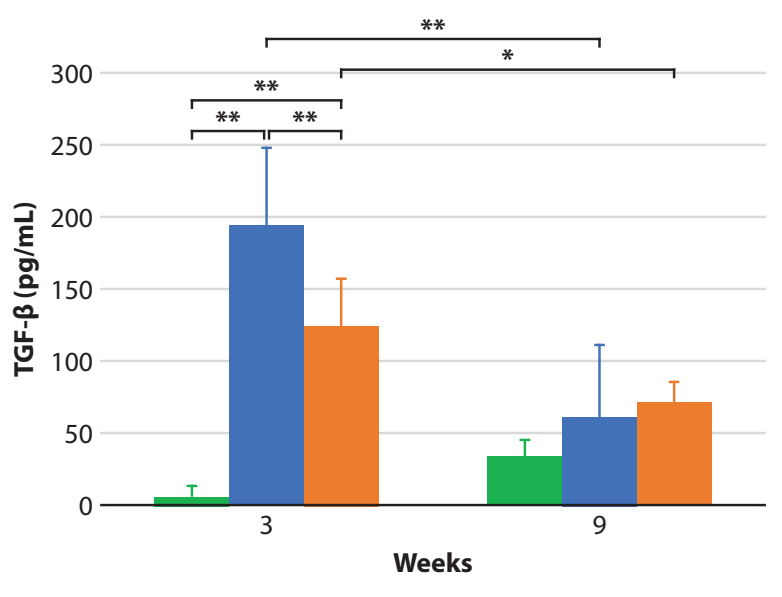

NC OVA without elastin OVA with elastin

Figure 5. Inhibitory effects on TGF- $\beta 1$ production by the neutralization of anti-elastin $\mathrm{Ab}$ following elastin intratracheal administration. TGF- $\beta 1$ levels in BALF at 3 weeks after OVA sensitization were significantly increased $(P<\mathbf{0 . 0 1})$ and were significantly reduced by the neutralization of anti-elastin $\mathrm{Ab}$ following elastin intratracheal administration $(P<0.01)$; however, there were no significant differences between the groups at week 9. TGF- $\beta 1$ levels in BALF at 9 weeks after OVA sensitization were significantly decreased in both groups without and with elastin $(P<0.01$ and $P<$ 0.05 , respectively) compared with at 3 weeks. The number of mice at each time point was $4 .{ }^{\star} P<0.05,{ }^{*} P<0.01$.

without elastin peptide $193.6 \pm 54.7 \mathrm{pg} / \mathrm{mL}$, with elastin peptide $123.6 \pm 33.6 \mathrm{pg} / \mathrm{mL}$; week 9: NC $32.8 \pm 12.6 \mathrm{pg} / \mathrm{mL}$, without elastin peptide $60.1 \pm 51.2 \mathrm{pg} / \mathrm{mL}$, with elastin peptide $70.5 \pm 14.8 \mathrm{pg} / \mathrm{mL})$. TGF- $\beta 1$ levels were significantly increased at week 3 and significantly decreased at week 9 in the OVA sensitization groups. 


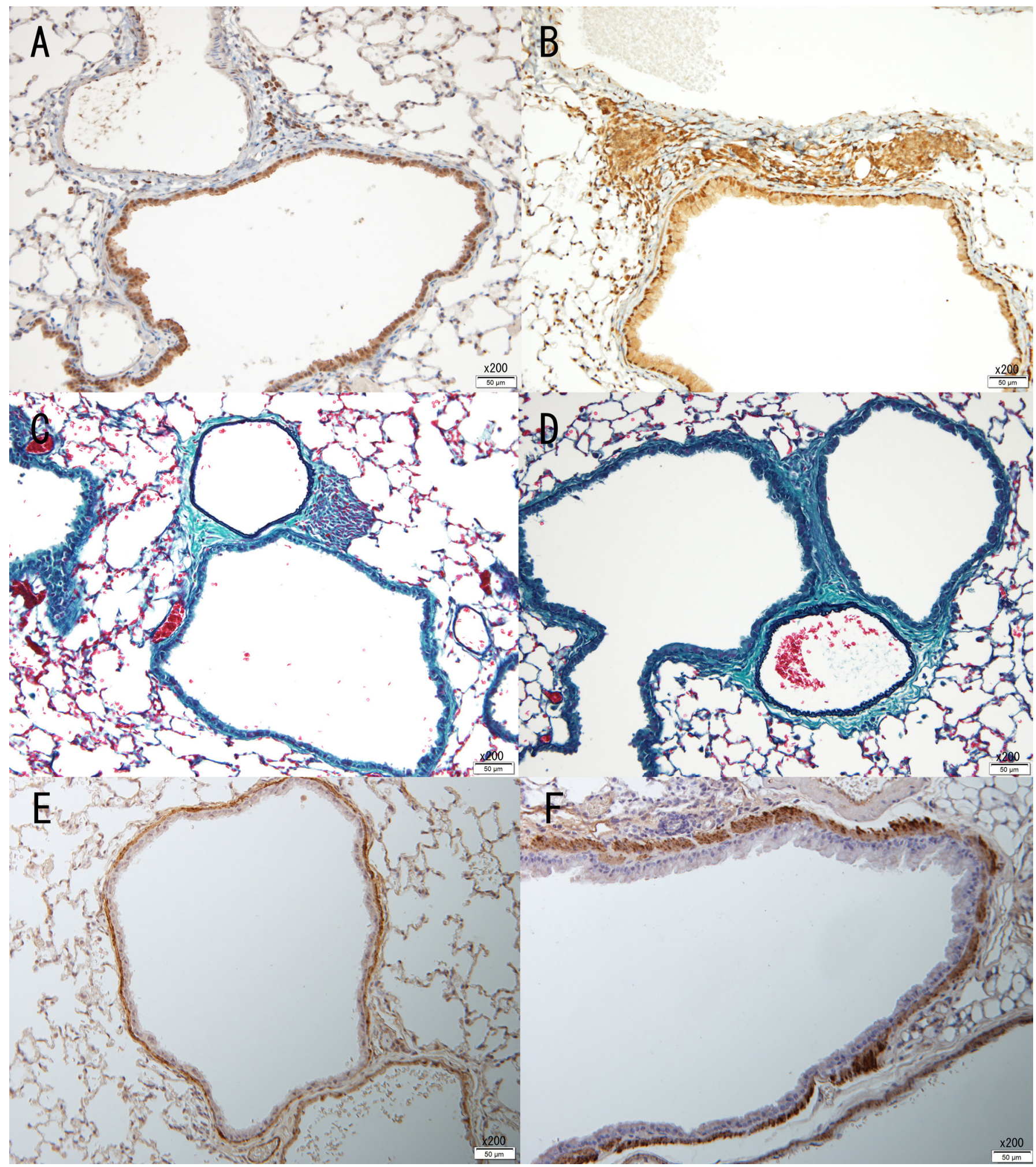

Figure 6. Inhibitory effects on airway remodeling via the neutralization of anti-elastin Ab following elastin intratracheal administration. Elastin expression at 9 weeks of OVA sensitization was significantly inhibited by neutralization (A) compared with non-neutralization (B). In the evaluation of fibrosis by elastic-Masson staining, fibrosis under the airway basement membrane at 9 weeks of OVA sensitization was significantly inhibited by neutralization (C) compared with non-neutralization (D). Peribronchial smooth muscle after 9 weeks of OVA sensitization was significantly inhibited by neutralization (E) compared with non-neutralization $(F)$. 


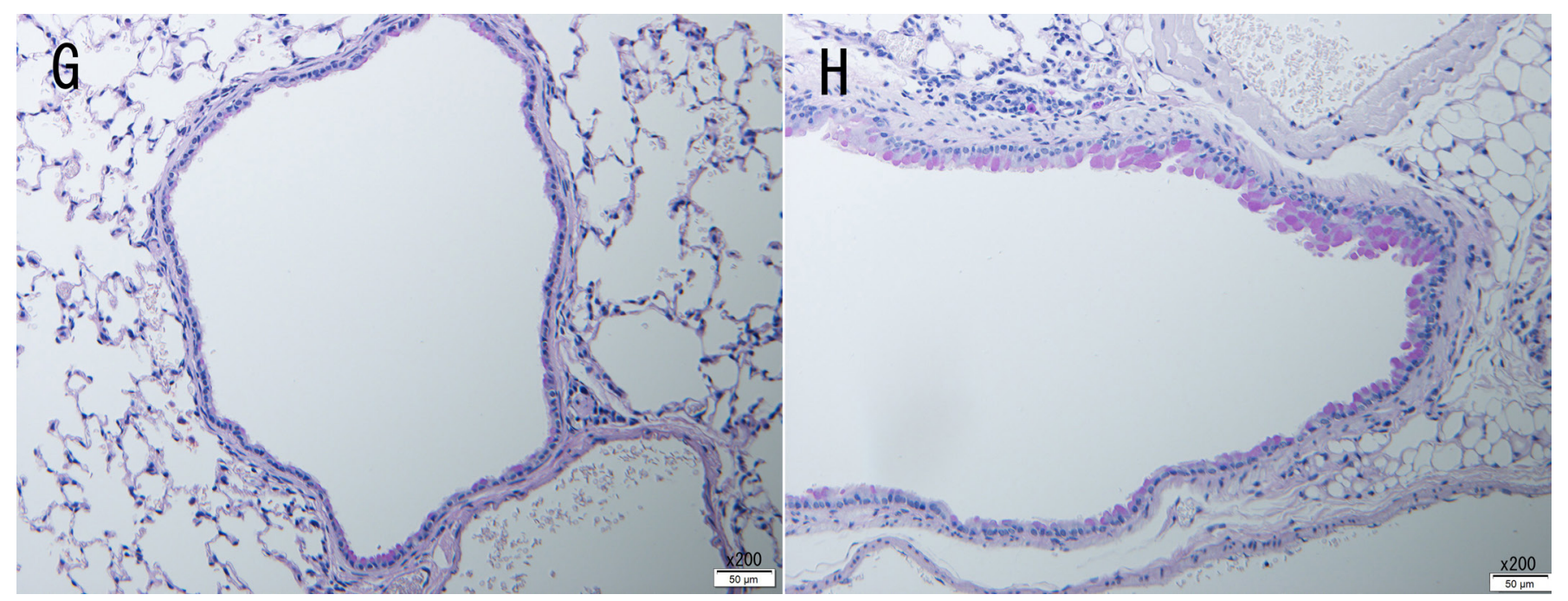

Figure 6. (Continued) In periodic acid-Schiff staining, mucosal goblet cells after 9 weeks of OVA sensitization were significantly inhibited by neutralization $(G)$ compared with non-neutralization $(H)$.

Elastin and fibrosis levels in lung tissues at 9 weeks after OVA sensitization are shown in Figure 6. Elastin expression was significantly inhibited by the neutralization of anti-elastin $\mathrm{Ab}$ following elastin intratracheal administration $(2.52 \pm$ $0.99 \%$ per field) compared with non-neutralization of anti-elastin $\mathrm{Ab}(7.13 \pm 3.51 \%$ per field $)(\mathrm{n}=3, P<0.01)$. Fibrosis under the airway basement membrane after 9 weeks of OVA sensitization was significantly inhibited by the neutralization of anti-elastin $\mathrm{Ab}$ following elastin intratracheal administration $(6.58 \pm 5.69 \%$ per field) compared with non-neutralization of anti-elastin $\mathrm{Ab}(14.29 \pm 31.10 \%$ per field) $(\mathrm{n}=$ $3, P<0.05)$. Peribronchial smooth muscle after 9 weeks of OVA sensitization was significantly inhibited by the neutralization of anti-elastin $\mathrm{Ab}(1.11 \pm 0.01 \%$ per field) compared with non-neutralization of anti-elastin $\mathrm{Ab}(2.22 \pm 0.47 \%$ per field) ( $\mathrm{n}=3 P<0.05)$. In periodic acid-Schiff staining, mucosal goblet cells after 9 weeks of OVA sensitization were significantly inhibited by the neutralization of anti-elastin $\mathrm{Ab}(0.40$ $\pm 0.09 \%$ per field) compared with non-neutralization of anti-elastin $\mathrm{Ab}(2.99 \pm 1.8 \%$ per field $)(\mathrm{n}=3, P<0.05)$.

\section{Discussion}

We defined the mouse model utilized in this study as an asthma model given that it has been used to observe airway hyperresponsiveness and acute exacerbation by OVA challenge in previous studies. ${ }^{9-11}$ In this model, anti-elastin $\mathrm{Ab}$ was recruited into the lungs through OVA sensitization, the same as eosinophils. Eosinophils, TGF- $\beta 1$ levels, and airway remodeling were reduced by the neutralization of anti-elastin $\mathrm{Ab}$. These results suggest that anti-elastin $\mathrm{Ab}$ may contribute to both eosinophilic inflammation and the progression of airway remodeling.

In other organs, elastin in serum has been found to be increased in patients with arteriosclerosis, ${ }^{12,13}$ and anti-elastin $\mathrm{Ab}$ has been shown to be increased in patients with symptomatic carotid stenosis. ${ }^{14}$ However, in other reports, anti-elastin $\mathrm{Ab}$ has been found to be lower in patients with arteriosclerosis and coronary artery disease. ${ }^{15,16}$ Moreover, anti-elastin $\mathrm{Ab}$ levels were found to be significantly higher in horses with moderate and severe arteriosclerosis compared with healthy horses. ${ }^{17}$ Thus, the role of anti-elastin $\mathrm{Ab}$ in arteriosclerosis, coronary artery disease, and COPD remains unclear.

In our previous study, anti-elastin $\mathrm{Ab}$ in sputum was elevated in patients with asthma. ${ }^{6}$ Elastin was expressed under the basement membrane around the bronchi, leading to airway remodeling. Patients with asthma showed stronger elastin expression compared with COPD patients or healthy participants. Although anti-elastin $\mathrm{Ab}$ in the lung will target elastin under the basement membrane around bronchi, it is not known if anti-elastin $\mathrm{Ab}$ is recruited in patients with asthma, and we could not find any reports of a relationship between anti-elastin $\mathrm{Ab}$ and asthma. As two contrary possibilities, anti-elastin Ab may be recruited to reduce elastin levels under the basement membrane around the bronchi to inhibit airway remodeling, or they may promote airway remodeling through a cascade of antigen-antibody reactions. Thus, we performed this study to clarify these hypotheses.

The peak level of anti-elastin $\mathrm{Ab}$ in the lung was detected at 3 weeks after sensitization, similar to the level of eosinophils, indicating that anti-elastin $\mathrm{Ab}$ in the lung was recruited in the early phase of allergic inflammation. Eosinophil infiltration and TGF- $\beta 1$ expression in the airway were inhibited by the neutralization of anti-elastin $\mathrm{Ab}$ in the early phase, leading to the inhibition of airway remodeling. In the relationship between eosinophils and TGF- $\beta 1$, the expression of TGF- $\beta$ in the lung was reduced by inhibiting eosinophils, ${ }^{18}$ and the number of eosinophils in BALF was reduced by anti-TGF- $\beta$ Ab in a mouse model of asthma, ${ }^{19}$ indicating that TGF- $\beta$ is involved in eosinophilic inflammation. Those reports suggest that eosinophilic inflammation may contribute to airway remodeling via TGF- $\beta$. However, it is possible that the progression of airway remodeling was inhibited by anti-elastin $\mathrm{Ab}$ directly, not via eosinophils or TGF- $\beta 1$. Further research is needed to clarify these pathways. 
We expected that anti-elastin $\mathrm{Ab}$ would be elevated in late-phase airway remodeling because elastin is a collagen fiber and because anti-elastin $\mathrm{Ab}$ may contribute to fibrosis. According to these results, anti-elastin $\mathrm{Ab}$ contributed to the production of airway remodeling, but not to the inhibition of airway remodeling. These results indicate that an elastin and anti-elastin $\mathrm{Ab}$ reaction recruits fibrotic cytokines such as TGF- $\beta$ and causes inflammation under the basement membrane around the bronchi. Consequently, fibrosis occurs under the basement membrane around the bronchi, leading to airway remodeling. In our mouse model of asthma, the peak level of anti-elastin Ab was detected at 3 weeks after OVA sensitization, and elastin under the basement membrane around bronchi was increased until 9 weeks. In this model, the airway inflammation that is induced by 2 or 3 weeks of OVA sensitization can subsequently be reduced by desensitization. $.^{9-11} \mathrm{Al}-$ though airway inflammation was reduced after 3 weeks, the results suggested that fibrosis continued as a wound healing response after severe inflammation and this led to airway remodeling. These results show that fibrosis under the basement membrane around the bronchi occurs in the early stage of allergic inflammation. We think that the inhibition of anti-elastin $\mathrm{Ab}$ in the early stage of asthma may be a useful treatment.

The increased proliferation of elastic fibers in the airway contributes to hyperresponsiveness and residual obstruction in the asthmatic airway. ${ }^{20,21}$ The exacerbation of asthma may contribute to the production of elastin because hypoxia increases elastin secretion from arterial smooth muscle cells. ${ }^{22}$ Thus, increased elastin levels may contribute to anti-elastin $\mathrm{Ab}$ production and promote airway remodeling. To inhibit airway remodeling, it is important to control asthma according to the Global Initiative for Asthma guidelines ${ }^{23}$ and to inhibit the increase of elastin under the basement membrane around the bronchi.

\section{Conclusion}

Anti-elastin $\mathrm{Ab}$ was recruited into the lungs by OVA sensitization in a mouse model of asthma. TGF- $\beta 1$ and eosinophils in BALF were reduced and airway remodeling was repressed via the inhibition of anti-elastin $\mathrm{Ab}$. The results suggest that anti-elastin $\mathrm{Ab}$ might be involved in airway remodeling and that inhibition of anti-elastin $\mathrm{Ab}$ may be a useful treatment for asthma.

\section{Acknowledgments}

We thank Ms. Ayako Yamamoto and Mr. Kazunori Fukuda of Dokkyo Medical University Saitama Medical Center for technical assistance. We also thank Ms. Seiko Sekiguchi and Ms. Akiko Maejima of Dokkyo Medical University Saitama Medical Center for assistance with preparing the manuscript.

\section{Conflict of interest statements}

The authors have no conflicts of interest to declare.

\section{Source of funding}

This study was supported by Dokkyo Medical University.

\section{Authors' contributions}

- TW and KS contributed to the conception and design of the study, data analysis, and interpretation of the data.

- ST, AH, NO, and KS contributed to the acquisition of data and data analysis.

- TO, HH, KK, MA, and YF contributed to interpretation of the data.

- All authors read and approved the final manuscript.

\section{References}

1. Brusselle GG, Joos GF, Bracke KR. New insights into the immunology of chronic obstructive pulmonary disease. Lancet. 2011;378:1015-26.

2. Bonarius HP, Brandsma CA, Kerstjens HA, Koerts JA, Kerkhof M, Nizankowska-Mogilnicka E, et al. Antinuclear autoantibodies are more prevalent in COPD in association with low body mass index but not with smoking history. Thorax. 2011;66:101-7.

3. Lee SH, Goswami S, Grudo A, Song LZ, Bandi V, Goodnight-White S, et al. Antielastin autoimmunity in tobacco smoking-induced emphysema. Nat Med. 2007;13:567-9.

4. Brandsma CA, Timens W, Geerlings M, Jekel H, Postma DS, Hylkema $\mathrm{MN}$, et al. Induction of autoantibodies against lung matrix proteins and smoke-induced inflammation in mice. BMC Pulm Med. 2010;10:64.

5. Wood AM, de Pablo P, Buckley CD, Ahmad A, Stockley RA. Smoke exposure as a determinant of autoantibody titre in al-antitrypsin deficiency and COPD. Eur Respir J. 2011;37:32-8.

6. Tokita S, Sugiyama K, Wakayama T, Arifuku H, Otsuji N, Sugitate K, et al. Elevation of anti-elastin antibody in patients with asthma. Asian Pac J Allergy Immunol. 2021 July 11. doi: 10.12932/AP-010221-1052. [Epub ahead of print].

7. Nakajima H, Iwamoto I, Tomoe S, Matsumura R, Tomioka H, Takatsu $\mathrm{K}$, et al. CD4+ Tlymphocytes and interleukin- 5 mediate antigen -induced eosinophil infiltration into the mouse trachea. Am Rev Respir Dis. 1992;146:374-7.

8. Tanaka H, Masuda T, Tokuoka S, Komai M, Nagao K, Takahashi Y, et al. The effect of allergen-induced airway inflammation on airway remodeling in a murine model of allergic asthma. Inflamm Res. 2001; 50:616-24.

9. Cheng G, Arima M, Honda K, Hirata H, Eda F, Yoshida N, et al. Anti-interleukin-9 antibody treatment inhibits airway inflammation and hyperreactivity in mouse asthma model. Am J Respir Crit Care Med. 2002;166:409-16.

10. Kumar RK, Herbert C, Foster PS. The "classical" ovalbumin challenge model of asthma in mice. Curr Drug Targets. 2008;9:485-94.

11. Obara K, Sugiyama K, Hirata H, Kikkawa Y, Sakio H, Arima M, et al. Monoclonal antibody against IL-5 receptor alpha, but not IL-5, inhibits airway hyperresponsiveness associated with airway remodeling. Int Med J. 2013;20:579-83.

12. Fülöp T Jr, Wei SM, Robert L, Jacob MP. Determination of elastin peptides in normal and arteriosclerotic human sera by ELISA. Clin Physiol Biochem. 1990;8:273-82.

13. Baydanoff S, Nicoloff G, Alexiev C. Age-related changes in the level of circulating elastin-derived peptides in serum from normal and atherosclerotic subjects. Atherosclerosis. 1987;66:163-8.

14. Tzvetanov P, Hegde V, Al-Hashel JY, Atanasova M, Sohal AP, Rousseff RT. Abnormal levels of serum anti-elastin antibodies in patients with symptomatic carotid stenosis. Clin Neurol Neurosurg. 2014;116:9-12.

15. Baydanoff S, Nicoloff G, Alexiev C. Age-related changes in anti-elastin antibodies in serum from normal and atherosclerotic subjects. Atherosclerosis. 1987;63:267-71.

16. Lee SH, Shin K, Park S, Kang SM, Choi D, Lee SH, et al. Circulating Anti-Elastin Antibody Levels and Arterial Disease Characteristics: Associations with Arterial Stiffness and Atherosclerosis. Yonsei Med J. 2015;56:1545-51.

17. De Keyser K, Berth M, Christensen N, Willaert S, Janssens S, Ducatelle $\mathrm{R}$, et al. Assessment of plasma anti-elastin antibodies for use as a diagnostic aid for chronic progressive lymphoedema in Belgian Draught Horses. Vet Immunol Immunopathol. 2015;163:16-22.

18. Flood-Page P, Menzies-Gow A, Phipps S, Ying S, Wangoo A, Ludwig MS, et al. Anti-IL-5 treatment reduces deposition of ECM proteins in the bronchial subepithelial basement membrane of mild atopic asthmatics J Clin Invest. 2003;112:1029-36. 
19. Bottoms SE, Howell JE, Reinhardt AK, Evans IC, McAnulty RJ. TGF- $\beta$ isoform specific regulation of airway inflammation and remodelling in a murine model of asthma. PLoS One. 2010;12;5:e9674.

20. Ingram JL, Slade D, Church TD, Francisco D, Heck K, Sigmon RW, et al. Role of Matrix Metalloproteinases-1 and -2 in Interleukin-13-Suppressed Elastin in Airway Fibroblasts in Asthma. Am J Respir Cell Mol Biol. 2016;54:41-50.

21. Setlakwe EL, Lemos KR, Lavoie-Lamoureux A, Duguay JD, Lavoie JP. Airway collagen and elastic fiber content correlates with lung function in equine heaves. Am J Physiol Lung Cell Mol Physiol. 2014;307:L252-60.
22. Kawakami S, Minamisawa S. Oxygenation decreases elastin secretion from rat ductus arteriosus smooth muscle cells. Pediatr Int. 2015;57: 541-5.

23. Global Initiative for Asthma [Internet]. Fontana: Global Initiative for Asthma; c2021 [cited 2021 Jan 15]. Global strategy for asthma management and prevention. Updated 2018; [about 1 screen]. Available from: https://ginasthma.org/wp-content/uploads/2019/01/2018-GINA. pdf. 